Pacific Journal of Mathematic 


\title{
EXTENSIONS OF REGULAR BOREL MEASURES
}

\author{
JACK HaRdy and H. Elton LaCEY
}

\begin{abstract}
This paper is concerned with the extension of regular Borel measures defined on the Borel sets generated by subtopologies of compact Hausdorff topologies.
\end{abstract}

Specifically, if $X$ is a nonempty set and $\tau$ is a topology on $X$, the Borel sets of $(X, \tau)$ are the members of the smallest $\sigma$-ring containing $\tau$. A regular Borel measure is taken to mean a finite-valued measure $\mu$ on the Borel sets of $(X, \tau)$ with property that

$$
\mu(B)=\sup \{\mu(F) \mid F \subseteq B, F \text { is closed }\}
$$

In this paper, the situation considered is the following: $\tau$ is a compact Hausdorff topology on $X$, and $\sigma$ is a regular (in the topological sense) sub-topology of $\tau$. The space $C(X, \tau)$ is the (partially ordered) Banach space of all continuous real-valued functions on $(X, \sigma)$, in the supremum norm. The space $C(X, \sigma)$ is similarly defined. By constructing a one-to-one correspondence between the collection of regular Borel measures on $(X, \sigma)$ and the collection of positive linear functionals on $C(X, \sigma)$ it is shown that every regular Borel measure on $(X, \sigma)$ can be extended to a regular Borel measure on $(X, \tau)$. This result is used to prove the existence of nonatomic regular Borel measures on compact Hausdorff spaces with perfect sub-sets.

The concept of a "partition space" plays a central role in this development.

Definition 1. Let $X$ be a topological space. A topological space $Y$ is said to be a partition space of $X$ if there is an onto function $f: X \rightarrow Y$ such that the topology for $X$ is the smallest topology for which $f$ is continuous.

A partition space is a special kind of quotient space [6:94]. Every topological space is a partition space of itself, and a partition space of a compact space is compact. It will be important to know when a topological space has a Hausdorff partition space. For a similar result about quotient spaces see [6:98]. A proof is given here because the notation of the proof is used later on.

THEOREM 2. A topological space $X$ has a Hausdorff partition space if and only if, for any two points $x$ and $y$ in $X$, if there is an open set $U$ such that $x \in U$ and $y \notin U$, then there are disjoint neighborhoods of $x$ and $y$. 
Proof. Let $Y$ be a Hausdorff partition space of $X$ and let $f: X \rightarrow Y$ be the function in Definition 1. Suppose $x$ and $y$ are two points of $X$, and $U$ is an open set such that $x \in U$ and $y \notin U$. There is an open set $E$ in $Y$ such that $U=f^{-1}(E)$. Then $f(x) \in E$ and $f(y) \notin E$. Thus $f(x) \neq f(y)$, and there are disjoint neighborhoods $E_{1}$ of $f(x)$ and $E_{2}$ of $f(y)$. Then $f^{-1}\left(E_{1}\right)$ and $f^{-1}\left(E_{2}\right)$ are disjoint neighborhoods of $x$ and $y$.

Conversely, for each $x \in X$, let $N_{X}$ be the set all elements $y \in X$ such that, for each open set $U, y \in U$ if and only if $x \in U$.

Let $Y=\left\{N_{X}: x \in X\right\}$. Define a function $f: X \rightarrow Y$ by $x \rightarrow N_{X}$ for every $x \in X$, and give $Y$ the largest topology for which $f$ is continuous (that is, a subset $B \subset Y$ is open if and only if $f^{-1}(B)$ is open). Then $Y$ is a Hausdorff partition space of $X$, because $f$ is certainly continuous, and if $U$ is open in $X$, then $U=\mathbf{U}\left\{N_{X}: x \in U\right\}$ implies $f^{-1}\left(N_{X}: x \in U\right)=U$.

COROLLARY 3. Every regular topological space has a Hausdorff partition space. In particular, every compact regular space has a compact Hausdorff partition space.

From now on, the "partition space" of a topological space $X$ will mean the partition space $Y=\left\{N_{X}: x \in X\right\}$ defined in the proof of Theorem 2.

The proof of the following theorem is straight-forward computation and hence will be ommited.

THEOREM 4. Let $Y$ be the partition space of a topological space $X$, and $B_{X}$ and $B_{Y}$ be the classes of Borel sets in $X$ and $Y$, respectively. Then

$$
B_{X}=\left\{f^{-1}(E): E \in B_{Y}\right\} .
$$

If $\mu$ and $\nu$ are real-valued functions on $B_{X}$ and $B_{Y}$ such that $\mu\left(f^{-1}(E)\right)=$ $\nu(E)$ for every $E \in B_{Y}$, then $\mu$ is a regular Borel measure on $X$ if and only if $\nu$ is a regular Borel measure on $Y$.

CoROLlary 5. In the notation of Theorem 4, to every regular Borel measure $\mu$ on $X$ assign a unique regular Borel measure $\nu_{\mu}$ on $Y$ by means of the formula

$$
\nu_{\mu}(E)=\mu\left(f^{-1}(E),\left(E \in B_{Y}\right) .\right.
$$

Then the mapping $\mu \rightarrow \nu_{\mu}$ is a one-to-one correspondence between the collection of regular Borel measures on $X$ and the collection of regular Borel measures on $Y$.

THEOREM 6. Let $Y$ be the partition space of a topological space 
$X$, and $C(X)$ and $C(Y)$ be the spaces of continuous real-valued functions on $X$ and $Y$, respectively. Then

$$
C(X)=\{g \circ f: g \in C(Y)\} \text {. }
$$

If $I(g \circ f)=J(g)$ for every $g \in C(Y)$, then $I$ is a positive linear functional on $C(X)$ if and only if $J$ is a positive linear functional on $C(Y)$.

Proof. Clearly $\{g \circ f: g \in C(Y)\} \subset C(X)$. On the other hand, take $h \in C(X)$. If $N_{X}$ is a fixed point of $Y$ and $y_{1}, y_{2} \in N_{X}$, then $h\left(y_{1}\right)=$ $h\left(y_{2}\right)$ (otherwise, there are disjoint neighborhoods $E_{1}$ and $E_{2}$ of $h\left(y_{1}\right)$ and $h\left(y_{2}\right)$, and $h^{-1}\left(E_{1}\right)$ and $h^{-1}\left(E_{2}\right)$ would be disjoint neighborhoods of $y_{1}$ and $\left.y_{2}\right)$. Thus $g\left(N_{x}\right)=h(x)$ defines a real-valued function $g$ on $Y$. Clearly $g \in C(Y)$ and $g \circ f=h$. This shows $C(X)=\{g \circ f: g \in C(Y)\}$.

The second part is immediate.

CoROLlary 7. In the notation of Theorem 6, to every positive linear functional $I$ on $C(X)$ assign a unique positive linear functional $J_{I}$ on $C(Y)$ by means of the formula

$$
J_{I}(g)=I(g \circ f),(g \in C(Y)) .
$$

Then the mapping $I \rightarrow J_{I}$ is a one-to-one correspondence between the collection of positive linear functionals on $C(X)$ and the collection of positive linear functionals on $C(Y)$.

Let $X$ be a compact regular space. To every regular Borel measure $\mu$ on $X$ assign a unique positive linear functional $I_{\mu}$ on $C(X)$ as follows. If $Y$ is the partition space of $X$, a regular Borel measure $\mu$ on $X$ gives rise (by Corollary 5) to a regular Borel measure $\nu$ on $Y$. Then the formula

$$
J_{\nu}(g)=\int_{Y} g d \nu \quad(g \in C(Y))
$$

defines a positive linear functional $J_{\nu}$ on $C(Y)$ which (by Corollary 7) defines a positive linear functional $I_{\mu}$ on $C(X)$.

THEOREM 8. For a compact regular space $X$, the mapping $\mu \rightarrow I_{\mu}$ is a one-to-one correspondence between the collection of regular Borel measures on $X$ and the collection of positive linear functionals on $C(X)$.

Proof. The Riesz Representation Theorem for compact Hausdorff spaces [4:177-178] shows that the mapping $\nu \rightarrow J_{\nu}$ is a one-to-one 
correspondence between the collection of regular Borel measures on $Y$ and the collection of positive linear functionals on $C(Y)$. Then Corollaries 5 and 7 complete the proof.

The next theorem (which generalizes a proof in [9]) is the main result of this paper.

THEOREM 9. Let $\tau$ be a compact Hausdorff topology for a set $X$, and let $\sigma$ be a regular topology for $X$ such that $\sigma \subset \tau$. Then every regular Borel measure on $(X, \sigma)$ can be extended to a regular Borel measure on $(X, \tau)$.

Proof. Let $\mu$ be a regular Borel measure on $(X, \sigma)$ and $I$ be the positive linear functional on $(X, \sigma)$ corresponding to $u$ by the mapping in Theorem 8. By [8, p. 18], $I$ can be extended to a positive linear functional $I^{*}$ on $C(X, \tau)$. Let $\mu^{*}$ be the regular Borel measure on $(X, \tau)$ such that

$$
I^{*}(g)=\int_{X} g d \mu^{*}
$$

for all $g \in C(X, \tau)$. It is shown that $\mu^{*}$ extends $\mu$.

Let $Y$ be the partition space of $(X, \sigma)$, and $\nu$ be the regular Borel measure on $Y$ defined by $\nu(E)=\mu\left(f^{-1}(E)\right)$ for every Borel set $E$ in $Y$.

Let $J$ be the positive linear functional on $C(Y)$ corresponding to $\nu$. Let $U$ be a member of $\sigma$. Then there is an open set $V$ in $Y$ such that $f^{-1}(V)=U$. Now, $\mu(U)=\nu(V)=$

$$
\begin{aligned}
& \sup \{J(h) \mid h \in C(Y), 0 \leqq h \leqq 1, h(y)=0 \quad \text { if } y \notin V\} \\
& =\sup \{I(k) \mid k \in C(X, \sigma), 0 \geqq k \leqq 1, k(x)=0 \quad \text { if } x \in U\} \\
& \leqq \sup \left\{I^{*}(k) \mid k \in C(X, \tau), 0 \leqq k \leqq 1, k(x)=0 \quad \text { if } x \notin U\right\} \\
& =\mu^{*}(U) \text {. }
\end{aligned}
$$

To show the reverse inequality, let $\varepsilon>0$ and let $K$ be a closed set in $(X, \tau)$ such that $K \subset U$ and $\mu^{*}(U)<\varepsilon+\mu^{*}(K)$. Since $(X, \sigma)$ is regular, for each $x$ in $k$, there is a set $V(x)$ such that $V(x)$ is closed in $(X, \sigma)$ and $x \in V(x) \subset U$. Since a compact regular space in normal [6:141], for each $x \in K$, there is a $g_{x} \in C(X, \sigma)$ such that $C H_{V(x)} \leqq g_{x} \leqq C H_{U}$ (CH is the characteristic function). Let $U(x)=$ $\left\{y \in X \mid g_{x}(y)+\varepsilon>1\right\}$. Then $\{U(x) \mid x \in K\}$ is a family of open subsets $q(X, \tau)$ which covers $K$, and there are $X_{1}, \cdots, X_{n}$ in $K$ such that $\left\{U\left(X_{i}\right) \mid i=1, \cdots, n\right\}$ covers $K$. Let $g=\max \left\{g_{x_{i}} \mid i=1, \cdots, n\right\}$. Then $g \in C(X, \sigma)$ and $\mu^{*}(U)<\varepsilon+\mu^{*}(K)=\varepsilon+\int_{X} C H_{K} d \mu^{*} \leqq \varepsilon+\int_{X}(g+\varepsilon) d \mu^{*}=$ $\varepsilon+\varepsilon \mu^{*}(X)+I^{*}(g) \leqq 2 \varepsilon+\mu(U)$ (since $\left.0 \leqq g \leqq C H_{U}\right)$. Thus $\mu^{*}(U)=$

${ }^{1}$ We wish to thank the referee for pointing out a simplification of the proof. 
$\mu(U)$ and by the regularity of $\mu, \mu^{*}$ extends $\mu$.

It is now shown how this can be applied to relationships between measures on $X$ and $Y$ and mappings from $X$ and $Y$. In particular, it is shown that if $X, Y$ are compact Hausdorff spaces and $f: X \rightarrow Y$ is a continuous onto map, then each regular Borel measure on $Y$ generates a regular Borel measure on $X$.

THEOREM 10. Let $X$ and $Y$ be a compact Hausdorff spaces, $f$ be a continuous function from $X$ onto $Y$, and $\nu$ be a regular Borel measure on $Y$. Then there is a regular Borel measure $\mu$ on $X$ such that

$$
\mu\left(f^{-1}(E)\right)=\nu(E)
$$

for every Borel set $E$ in $Y$. Moreover, if $\nu$ is nonatomic, then so is $\mu$.

Proof. Let $\tau$ denote the topology for $X$, and let

$$
\sigma=\left\{f^{-1}(U): U \text { open in } Y\right\} \text {. }
$$

Then $\sigma$ is a regular topology for $X, \sigma \subset \tau, C(X, \sigma)$ is a linear subspace of $C(X, \tau)$, and $C(X, \sigma)$ contains the constant functions. Thus Theorem 9 implies that every regular Borel measure on $(X, \sigma)$ can be extended to a regular Borel measure on $(X, \tau)$.

For every Borel set $E$ in $Y$, define $\mu_{0}\left(f^{-1}(E)\right)=\nu(E)$. The proof of Theorem 4 shows that $\mu_{0}$ is a regular Borel measure on $(X, \sigma)$. Let $\mu$ be a regular Borel measure on $(X, \tau)$ which extends $\mu_{0}$. Then $\mu\left(f^{-1}(E)\right)=\nu(E)$ for every Borel set $E$ in $Y$. If $\nu$ is nonatomic, then, for every $x \in X$,

$$
0 \leqq \mu(x) \leqq \mu\left(f^{-1} f(x)\right)=\nu(f(x))=0
$$

implies $\mu(x)=0$, and thus $\mu$ is nonatomic.

CoROLlaRY 11. [9]. If $X$ is a compact Hausdorff space with a nonempty perfect set, then there is a nonzero, nonatomic regular Borel measure on $X$.

Proof. There is a continuous map of $X$ onto [0,1]. Thus, in Theorem 10 one can use Lebesgue measure for $\nu$.

Corollary 12. Let $X$ be a compact Haudorff space, $\left\{P_{n}\right\}$ be a disjoint sequence of perfect subsets of $X$, and $\left\{a_{n}\right\}$ be a sequence of nonnegative real numbers such that $\sum a_{n}<\infty$. Then there is a nonatomic regular Borel measure $\mu$ on $X$ such that $\mu\left(P_{n}\right)=a_{n}$ for every $n$. 
Proof. For each $n$, let $\nu_{n}$ be a nonatomic regular Borel measure on $P_{n}$ such that $\nu_{n}\left(P_{n}\right)=a_{n}$, and define $\mu_{n}(E)=\nu_{n}\left(E \cap P_{n}\right)$ for every Borel set $E$ in $X$. Then $\mu=\sum \mu_{n}$ is a nonatomic regular Borel measure on $X$, and $\mu\left(P_{n}\right)=a_{n}$ for every $n$.

For the last theorem some additional terminology is needed. Let $X$ and $Y$ be compact Hausdorff spaces. By $M(X)$ is meant the Banach lattice of all regular Borel measures on $X$ under the total variation norm. Of course, $M(X)$ is precisely the Banach space dual of $C(X)$. If $\nu$ is a regular Borel measure on $Y$, by $L^{1}(\nu)$ is meant the Banach lattice of all $\nu$-integrable functions on $Y$, under the integral norm.

THEOREM 13. If there is a continuous map $f$ of $X$ onto $Y$, then $L^{1}(\nu)$ is linearly isometric to a subspace of $M(X)$.

Proof. Let $\mu$ be the regular Borel measure associated with $\nu$ of Theorem 11. Let $N$ be the normed linear space whose elements are the continuous functions on $Y$, but whose norm is the integral norm with respect to $\nu$. Then $N$ is dense in $L^{1}(\nu)$. Define the linear operator $A: N \rightarrow M(X)$ by $(A g)(h)=\int_{X} h(g \circ f) d \mu$ for all $g \in N, h \in C(X)$. Now, $\|A g\|=\int_{X}|g \circ f| d \mu=\|g\|$ and $A$ is an isometry of $N$ into $M(X)$. Since $N$ is dense in $L^{1}(\nu), A$ can be uniquely extended to an isometry of $L^{1}(\nu)$ into $M(X)$.

\section{BIBLIOGRAPHY}

1. S. K. Berberian, Measure and Integration, MacMillian, New York, 1965.

2. R. B. Darst, Perfect null sets in compact Hausdorff spaces, Proc. Amer. Math. Soc. 16 (1965), 845.

3. P. R. Halmos, Measure Theory, D. Van Nostrand, Princeton, New Jersey, 1950.

4. E. Hewitt and K. Stromberg, Real and Abstract Analysis, Springer-Verlag, New York, 1965.

5. S. Kaplan, On the second duals of the space of cotinuous functions (I), Trans. Amer. Math. Soc. 86 (1957), 70-90.

6. J. L. Kelley, General Topology, D. Van Nostrand, Princeton, New Jersey, 1955.

7. - Measures on Boolean algebras, Pacific J. Math. 9 (1959), 1165-1177.

8. J. L. Kelley and I. Namioka, Linear Topological Spaces, D. Van Nostrand, Princeton, New Jersey, 1963.

9. A. Pelczynski and Z. Semadeni, Spaces of continuous functions (III), Studia Math. 18 (1959), 211-222.

Received July 10, 1967. The second author held a Resident Post-Doctorial Research Associateship at NASA-MSC Houston, Texas during the preparation of part of this paper.

The University of TeXas at Austin 


\title{
PACIFIC JOURNAL OF MATHEMATICS
}

\author{
EDITORS
}

\section{H. ROYDEN}

Stanford University

Stanford, California

\section{J. P. JANS}

University of Washington Seattle, Washington 98105

\section{J. DugundJI}

Department of Mathematics

Rice University

Houston, Texas 77001

RICHARD ARENS

University of California

Los Angeles, California 90024

\section{ASSOCIATE EDITORS}

E. F. BECKENBACH
F. WOLF

K. YosidA

\section{SUPPORTING INSTITUTIONS}

\author{
UNIVERSITY OF BRITISH COLUMBIA \\ CALIFORNIA INSTITUTE OF TECHNOLOGY \\ UNIVERSITY OF CALIFORNIA \\ MONTANA STATE UNIVERSITY \\ UNIVERSITY OF NEVADA \\ NEW MEXICO STATE UNIVERSITY \\ OREGON STATE UNIVERSITY \\ UNIVERSITY OF OREGON \\ OSAKA UNIVERSITY \\ UNIVERSITY OF SOUTHERN CALIFORNIA
}

\author{
STANFORD UNIVERSITY \\ UNIVERSITY OF TOKYO \\ UNIVERSITY OF UTAH \\ WASHINGTON STATE UNIVERSITY \\ UNIVERSITY OF WASHINGTON \\ MERICAN MATHEMATICAL SOCIETY \\ CHEVRON RESEARCH CORPORATION \\ TRW SYSTEMS \\ NAVAL ORDNANCE TEST STATION
}

Mathematical papers intended for publication in the Pacific Journal of Mathematics should be in typed form or offset-reproduced, double spaced with large margins. Underline Greek letters in red, German in green, and script in blue. The first paragraph or two must be capable of being used separately as a synopsis of the entire paper. It should not contain references to the bibliography. Manuscripts may be sent to any one of the four editors. All other communications to the editors should be addressed to the managing editor, Richard Arens, University of California, Los Angeles, California 90024.

Each author of each article receives 50 reprints free of charge; additional copies may be obtained at cost in multiples of 50 .

The Pacific Journal of Mathematics is published monthly. Effective with Volume 16 the price per volume (3 numbers) is $\$ 8.00$; single issues, $\$ 3.00$. Special price for current issues to individual faculty members of supporting institutions and to individual members of the American Mathematical Society: $\$ 4.00$ per volume; single issues $\$ 1.50$. Back numbers are available.

Subscriptions, orders for back numbers, and changes of address should be sent to Pacific Journal of Mathematics, 103 Highland Boulevard, Berkeley 8, California.

Printed at Kokusai Bunken Insatsusha (International Academic Printing Co., Ltd.), 7-17, Fujimi 2-chome, Chiyoda-ku, Tokyo, Japan.

PUBLISHED BY PACIFIC JOURNAL OF MATHEMATICS, A NON-PROFIT CORPORATION

The Supporting Institutions listed above contribute to the cost of publication of this Journal, but they are not owners of publishers and have no responsibility for its content or policies. 


\section{Pacific Journal of Mathematics}

\section{Vol. 24, No. $2 \quad$ June, 1968}

John Suemper Alin and Spencer Ernest Dickson, Goldie's torsion theory and its derived functor . . . . . . . . . . . . . . . . . . . . . . . . . . . 195

Steve Armentrout, Lloyd Lesley Lininger and Donald Vern Meyer,

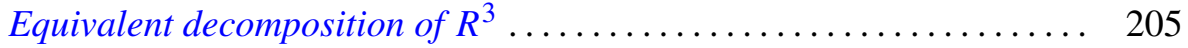

James Harvey Carruth, A note on partially ordered compacta ........... 229

Charles E. Clark and Carl Eberhart, A characterization of compact

connected planar lattices........................... 233

Allan Clark and Larry Smith, The rational homotopy of a wedge ......... 241

Donald Brooks Coleman, Semigroup algebras that are group algebras .... 247

John Eric Gilbert, Convolution operators on $L^{p}(G)$ and properties of

locally compact groups ............................. 257

Fletcher Gross, Groups admitting a fixed-point-free automorphism of order

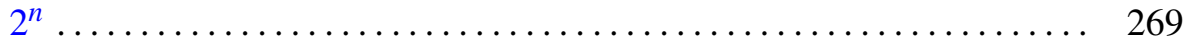

Jack Hardy and Howard E. Lacey, Extensions of regular Borel measures . . . 277

R. G. Huffstutler and Frederick Max Stein, The approximation solution of

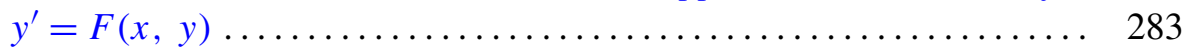

Michael Joseph Kascic, Jr., Polynomials in linear relations . . . . . . . . . . 291

Alan G. Konheim and Benjamin Weiss, A note on functions which

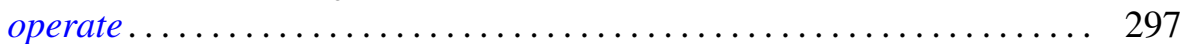

Warren Simms Loud, Self-adjoint multi-point boundary value problems ... 303 Kenneth Derwood Magill, Jr., Topological spaces determined by left ideals

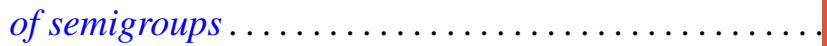

Morris Marden, On the derivative of canonical products . . .

J. L. Nelson, A stability theorem for a third order nonlinear differential

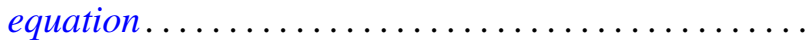

Raymond Moos Redheffer, Functions with real poles and zeros ...

Donald Zane Spicer, Group algebras of vector-valued functions ...

Myles Tierney, Some applications of a property of the functor $E f$ 